\title{
TERAPIA DE RESGATE COM RITUXIMAB NA RECIDIVA DA GLOMERULOESCLEROSE SEGMENTAR E FOCAL (GESF) PÓS-TRANSPLANTE: RELATO DE CASO.
}

\author{
Rituximab for post-transplantation Focal Segmental Glomerulosclerosis (FSGS) relapse: a case report
}

\author{
Luciana Tanajura Santamaria Saber, Camila Bastos, Ricardo Araújo Mothé, Roberto Silva Costa, Valmir Aparecido Muglia, \\ Mariana Arreaza Giovannetti, Tatiana Silva Lima.
}

\begin{abstract}
RESUMO
Após um primeiro transplante renal, 20\% a 40\% das Glomerulonefrites esclerosantes segmentar e focal (GESF) recidivam, frequentemente levando à perda do enxerto. Esses dados podem aumentar em mais de $80 \%$ em casos de pacientes recebendo um segundo transplante após a recidiva inicial. Apesar de geralmente ineficaz, a plasmaferese é ainda considerada como a primeira opção terapêutica para casos de recidiva de GESF pós-transplante. Recentemente, o anticorpo monoclonal anti-CD20 Rituximab (RTX) tem surgido como alternativa terapêutica para esses casos. Relatamos o caso de um paciente do sexo masculino de 34 anos de idade portador de insuficiência renal crônica secundária a glomerulonefrite não biopsiada, tendo sido submetido a transplante renal de doador falecido. Cerca de 40 dias após o transplante, o paciente apresentou síndrome nefrótica, que foi diagnosticada como GESF. O paciente foi submetido a cinco sessões de plasmaferese sem resposta. Posteriormente, foi tratado com duas doses endovenosas de RTX $375 \mathrm{mg} / \mathrm{m}^{2}$, com sete dias de intervalo entre as doses, e uma terceira dose 60 dias após a segunda dose. Essa estratégia permitiu obter remissão completa e sustentada da síndrome nefrótica e excelente função renal, que se mantém até o momento, um ano após o tratamento.
\end{abstract}

Descritores: Glomerulosclerose Segmentar e Focal; Recidiva; Tratamento; Plasmaferese, Transplante de Rim.

Instituição:

Serviço de Transplante Renal, Hospital das Clinicas da FMRP-USP, Ribeirão Preto/SP.

\section{Correspondência:}

Luciana Tanajura Santamaria Saber

Serviço de Transplante Renal do HCFMUSP - Campus Universitário - Monte Alegre, CEP 14048-900, Ribeirão Preto/SP

Tel: (16) 3602-2225 Fax: (16) 3633-2189

E-mail: lucianasaber@gmail.com

\section{INTRODUÇÃO}

Glomerulonefrite esclerosante segmentar e focal (GESF), tanto como doença renal primária ou secundária é a causa mais frequente de síndrome nefrótica em adultos e uma das patologias mais comuns que recidivam após o transplante. As taxas de recidiva são estimadas em $20-40 \%$ após um primeiro transplante renal e geralmente ocorrem dentro do primeiro mês, sendo caracterizadas pelo aparecimento de proteinúria maciça e geralmente levam à perda do enxerto. Uma vez que ocorre a recidiva, cerca de $30 \%$ dos pacientes progridem para insuficiência renal crônica em cinco anos ${ }^{1,2}$ e o risco de recidiva aumenta para $80 \%$ nos transplantes posteriores. ${ }^{3}$ Até o momento não há consenso para o tratamento da recidiva, mas, apesar de frequentemente ineficaz, a plasmaferese é ainda considerada como a primeira opção no tratamento das recidivas. ${ }^{4}$ Recentemente, o anticorpo monoclonal anti-CD20 Rituximab tem surgido como outra opção. Rituximab foi utilizado inicialmente em alguns pacientes que desenvolveram doença linfoproliferativa pós-transplante (PTLD) e que também apresentavam recidiva precoce de GESF. Houve remissão da PTLD e, como efeito colateral, houve também diminuição significativa dos níveis de proteinúria. ${ }^{5,6}$ Neste trabalho, relatamos um caso de remissão sustentada de síndrome nefrótica causada pela recidiva de GESF após um primeiro transplante renal. 


\section{RELATO DE CASO}

S.D.C., sexo masculino, 34 anos, portador de insuficiência renal crônica secundária a glomerulonefrite crônica não biopsiada foi submetido a transplante renal com doador falecido, em 28 de outubro de 2008. Compatibilidade HLA foi A1B1DR1, atividade de anticorpos contra painel de zero, tempo de isquemia fria de 35 horas e imunossupressão inicial consistiu de Basiliximab (duas doses de 20mg), Tacrolimus, Micofenolato Sódico e esteróides. Paciente evoluiu inicialmente com Necrose Tubular Aguda, seguida de Rejeição Aguda Banff 4I a, tratada com pulso de Metilprednisolona (12-14 de Novembro), com boa resposta. Cerca de 40 dias após o transplante, paciente apresentou síndrome nefrótica acompanhada de alteração da função renal. Paciente foi então submetido à biopsia renal, a qual foi inconclusiva; a segunda biopsia revelou tecido normal, com imunofluorescência negativa. Paciente desenvolveu proteinúria maciça, apesar da introdução de inibidores de enzima de conversão da angiotensina (iECA). Nesse meio tempo, o paciente foi convertido de Micofenolato Sódico para Azatioprina, devido à intolerância gastrintestinal. Foi realizada então uma terceira biopsia, que revelou a presença de GESF (Figura 1). Iniciou-se tratamento com plasmaferese, e após cinco sessões de plasmaferese sem resposta (realizadas em dias alternados, pelo período de dez dias), optamos por administrar duas doses endovenosas de Rituximab (RTX) $375 \mathrm{mg} / \mathrm{m}^{2}$, com um intervalo de sete dias entre as doses.

Figura 1: Lesão de esclerose segmentar com células espumosas e hipertrofia podocitária (Tricrômico de Masson. Objetva: 40x).

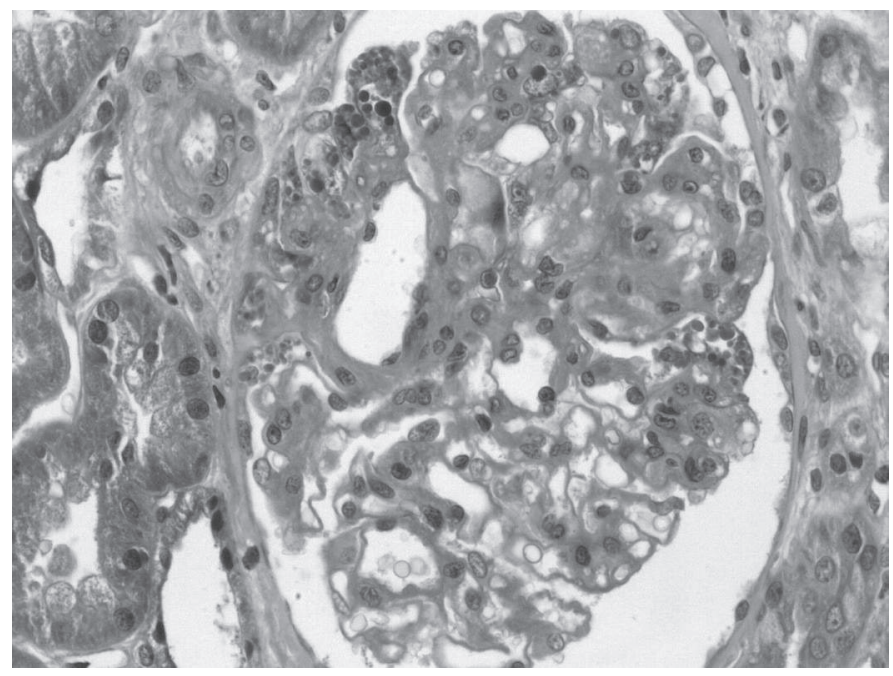

Os níveis de proteinúria caíram de $20,1 \mathrm{~g} /$ dia para $5,8 \mathrm{~g} / \mathrm{dia}$, assim como os níveis de creatinina, de $2,1 \mathrm{mg} \%$ para $1,5 \mathrm{mg} \%, 14$ dias após a segunda dose. Losartan foi adicionado juntamente com dose máxima de iECA, tendo havido estabilização dos níveis de proteinúria ao redor de $3,5 \mathrm{~g} / \mathrm{dia}$. Sessenta dias após a segunda dose de RTX, uma terceira dose de RTX $375 \mathrm{mg} / \mathrm{m}^{2}$ foi administrada. Quatorze dias após a terceira dose, a proteinúria diminuiu ainda mais, para níveis abaixo de $1.0 \mathrm{~g} /$ dia e a creatinina ficou estável em torno de 1,4 mg\% (Figura 2). A não ser por discreta trombocitopenia, não houve eventos adversos significativos, relacionados ao uso de RTX.
Figura 2: Proteinúria de 24 horas (____-) e creatinina sérica (........) em diferentes momentos após o transplante e intervenções terapêuticas.

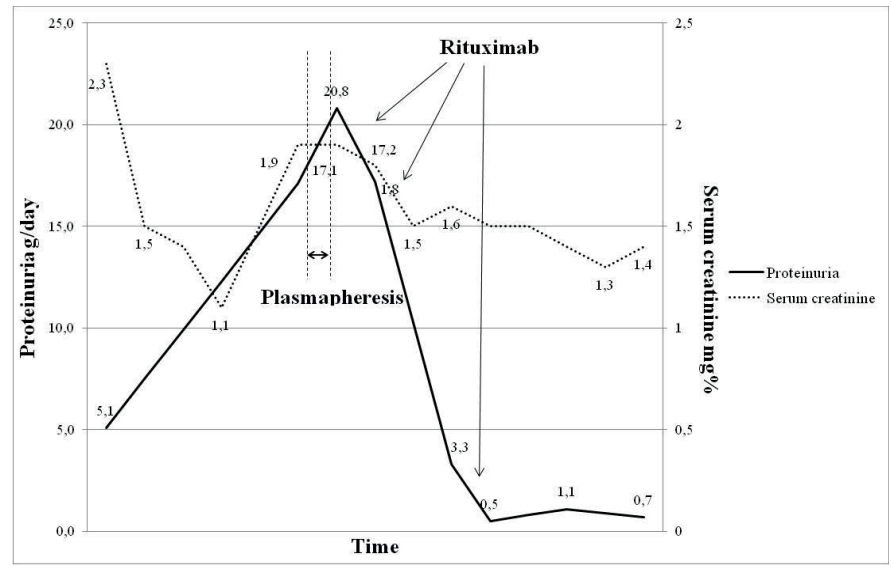

Essa estratégia terapêutica permitiu obter evidente remissão significativa e sustentada da síndrome nefrótica, além de excelente função renal, a qual persiste até o momento, um ano após o tratamento com RTX (a terceira e última dose foi administrada em 21 de Agosto de 2009): exames de outubro 2010 - creatinina $1.26 \mathrm{mg} \%$, proteinúria de 24 horas: $628 \mathrm{mg}$. Paciente também não apresentou nenhum evento infeccioso importante neste período.

\section{DISCUSSÃO}

A recidiva de GESF após transplante renal, além da morbidade decorrente da própria síndrome nefrótica, pode levar à perda acelerada do enxerto e até hoje muito se debate sobre a existência ou não de fatores de risco identificáveis que possam prever a sua recorrência. Até o momento, alguns fatores têm sido relatados: nefrectomia pré-transplante, idade do doador maior que 43 anos, transplante com doador vivo relacionado, receptor feminino, curto intervalo de tempo entre o diagnóstico inicial de GESF e o início da diálise e níveis altos de proteinúria pré-transplante. ${ }^{7}$

A patogênese, tanto da doença original como de sua recidiva, não foi ainda completamente elucidada. ${ }^{1}$ A presença de um fator circulante, que pode aumentar a permeabilidade glomerular à albumina, tem sido descrito em alguns pacientes, ${ }^{8,9}$ o que levou, portanto, a estudos com plasmaferese e/ou imuno-adsorção de proteína A para os casos de recidiva de GESF, mas a resposta parece ser variável e imprevisível. ${ }^{4,7}$

No caso aqui relatado, não sabemos qual a glomerulonefrite primária que levou à insuficiência renal crônica, mas considerando-se a evolução, muito provavelmente foi GESF. Infelizmente, as duas primeiras biopsias realizadas quando do aparecimento da síndrome nefrótica falharam na detecção do diagnóstico de GESF, o que levou a um atraso no início do tratamento. Apesar de tardias, as sessões de plasmaferese foram realizadas, sem qualquer resposta, o que nos levou a procurar por uma terapêutica alternativa. Tanto a função renal como a síndrome nefrótica per se estavam piorando quando o tratamento com RTX foi iniciado. Esse foi realmente um momento de virada na evolução do paciente, a partir do qual sua condição melhorou progressivamente. Apesar de ao menos teoricamente podermos considerar a hipótese de resposta tardia à plasmaferese, não acreditamos nessa possibilidade, uma vez que não houve sequer 
esboço de qualquer resposta; ao contrário: notamos apenas piora clinica e laboratorial até a introdução do Rituximab.

Rituximab é um anticorpo monoclonal específico para linfócitos B (antiCD20), que tem sido utilizado no tratamento de diversas patologias de etiologia imunológicas. Seu efeito na atividade da doença parece ser tempo-limitado e ainda não há um consenso de como dever ser utilizado.

Nós quisemos consolidar a resposta obtida e, portanto, decidimos por administrar uma terceira dose sessenta dias após a segunda dose, o que se mostrou extremamente benéfico.

Até o momento, apenas 14 estudos foram publicados, relatando tratamentos de recidiva de GESF pós-transplante renal com RTX, envolvendo um total de 26 pacientes, a maioria dos quais descrita por Terje Apeland e Anders Hartmann.

5,6,10-21 Até onde sabemos, este é o $15^{\circ}$ caso apresentando apenas GESF (sem PTLD associado) que respondeu com remissão significativa e sustentada da síndrome nefrótica. Por outro lado, vale a pena ressaltar que até o momento, contamos apenas com relatos de casos, não havendo nenhum estudo clinico randomizado, fazendo com que haja um viés nos resultados, uma vez que apenas resultados positivos são publicados. Faz-se necessária então a realização de estudos amplos e randomizados.

Em conclusão, Rituximab parece constituir uma arma terapêutica eficaz para recidiva pós-transplante de GESF, especialmente para aqueles pacientes que não respondem à plasmaferese.

\section{ABSTRACT}

After a first renal transplantation, $20 \%$ to $40 \%$ of Focal Segmental Glomerulosclerosis (FSGS) relapse, frequently leading to renal loss. These data can increase in more than $80 \%$ of patients receiving a second transplant after a recurrence. Although often ineffective, plasmapheresis is still considered to be the first option to treat relapses. Recently, anti-CD-20 monoclonal antibody Rituximab (RTX) has appeared as an alternative choice. We report a case of a 34 year old male patient who was submitted to a kidney transplant from deceased donor due to end stage renal disease secondary to a non-biopsied glomerulonephritis. About 40 days after transplant he presented nephrotic syndrome, diagnosed as FSGS. He underwent five plasmapheresis sessions with no response. Then, he received two intravenous doses of RTX $375 \mathrm{mg} / \mathrm{m} 2$, with a seven day interval between doses and a third dose 60 days after the second one. This strategy allowed attaining sustained full remission of the nephrotic syndrome and excellent graft function, which persists one year after RTX treatment.

Keywords: Glomerulosclerosis, Focal Segmental; Recurrence; Treatment; Plasmapheresis, Kidney Transplantation.

\section{REFERÊNCIAS}

1. Meyrier A. Mechanisms of disease: focal segmental glomerulosclerosis. Nat Clin Pract Nephrol. 2005;1:44.

2. Floege J. Recurrent glomerulonephritis following renal transplantation: an update. Nephrol Dial Transplant. 2003;18(7):1260-5.

3. Newstead CG. Recurrent disease in renal transplants. Nephrol Dial Transplant. 2003;18(S6):68

4. KDIGO 2010 ( Clinical Practice Guideline for the care of Kidney Transplant Recipients; www.kdigo.org )

5. Nozu K, Iijima K, Fujisawa M et al. Rituximab treatment for post-transplant lymphoproliferative disorder (PTLD) Induces complete remission of recurrent nephritic syndrome. Pediatr Nephrol. 2005;20:1660-3.

6. Pescovitz MD, Book BK, Sidner RA. Resolution of recurrent focal segmental glomerulosclerosis proteinuria after Rituximab treatment. N Engl J Med. 2006;354:1961-3.

7. Sener A, Bella AJ, Nguan C et al. Focal segmental glomerular sclerosis in renal transplant recipients: predicting early disease recurrence may prolong allograft function. Clin Transplant. 2009;23:96-100.

8. Herve C, Dantal J. Possible new perspectives for our understanding of nephrotic syndrome recurrence. Nephrol Dial Transplant. 2006;21:10

9. Vicenti F, Ghigger GM. New insights into the pathogenesis and the therapy of recurrent focal glomerulosclerosis. Am J Transplant. 2005;5:1179.

10. Grossmann J, Scheuermann E-H, Porubsky S et al. Abrogation of nephrotic proteinuria by rituximab treatment in a renal transplant patient with relapsed focal segmental glomerulosclerosis. Transpl Int. 2007;20(6):558-62.

11. Hristea D, Hadaya K, Marangon N et al. Successful treatment of recurrent focal segmental glomerulosclerosis after kidney transplantation by plasmapheresis and
Rituximab. Transplant Int. 2007;20(1):102-5.

12. El-Firjani A, Hoar S, Karpinski J et al. Post-transplant focal segmental glomerulosclerosis refractory to plasmapheresis and Rituximab therapy. Nephrol Dial Transplant. 2008;23(1):425.

13. Kamar N, Faguer S, Esposito L et al. Treatment of focal segmental glomerular sclerosis with Rituximab: 2 cases reports. Clin Nephrol. 2007;67:250-4.

14. Marks SD, McGraw M. Does Rituximab treat recurrent focal segmental glomerulosclerosis post-renal transplantation? Pediatr Nephrol. 2007;22:158-60.

15. Meyer TN, Thaiss F, Stahl RA. Immunoadsorbtion and Rituximab therapy in a second living-related kidney transplant patient with recurrent focal segmental glomerulosclerosis. Transpl Int. 2007;20:1066-71.

16. Nakayama M, Kamei K, Nozu K et al. Rituximab for refractory focal segmental glomerulosclerosis. Pediatr Nephrol. 2008;23:481-5.

17. Yabu JM, Ho B, Scanding JD et al. Rituximab failed to improve nephrotic syndrome in renal transplant patients with recurrent focal segmental glomerulosclerosis. Am J Transplant. 2008;8:222-7.

18. Apeland T, Hartmann A. Rituximab therapy in early recurrent focal segmental sclerosis after renal transplantation. Nephrol Dial Transplant. 2008;23:2091-4.

19. Rodriguez-Ferrero M, Ampuero J, Anaya F. Rituximab and chronic plasmapheresis therapy of nephrotic syndrome in renal transplantation patients with recurrent focal segmental glomerulosclerosis. Transplant Proc. 2009;41(6):2406-8

20. Dello Strologo L, Guzzo I, Laurenzi C et al. Use of Rituximab in focal glomerulosclerosis relapses after renal transplantation. Transplantation. 2009;88(3):417-20

21. Bayrakci US, Baskin E, Skalli H et al. Rituximab for post-transplant recurrences of FSGS. Pediatr Transplant. 2009;13(2):240-3. 